CERN LIBRARIES, GENEVA

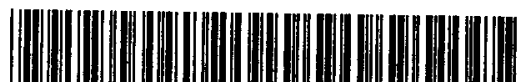

SCAN-9506042

$$
\operatorname{Sin} 9524
$$

NORDITA - 95/40 A

CLASSICAL AND QUANTUM WORMHOLES WITH

PERFECT FLUIDS AND SCALAR FIELDS

A. Carlini ${ }^{\diamond}$ D.H. Coule* D.M. Solomons*

$\diamond$ Nordita, Blegdamsvej 17, DK-2100 Copenhagen $\varnothing$, Denmark.

*Department of Applied Mathematics, University of Cape Town, Rondebosch 7700, South Africa.

NORDITA - Nordisk Institut for Teoretisk Fysik

Blegdamsvej 17 DK-2100 København Ø Danmark 


\section{Classical and quantum wormholes with perfect fluids and scalar fields}

\author{
A. Carlini $\diamond 1$ D.II. Coule ${ }^{* 2}$ and D.M. Solomons $* 3$
}

$\diamond$ NORDITA, Blegdamsvej 17, DK-2100 Copenhagen (). Denmark *Department of Applied Mathematics, University of ('ape 'Town. Rondelosch 7700 , South Africa

\section{Abstract}

Wormholes are obtained by the analytic continuation of closed recollapsing FRW universes. We demonstrate this for a perfect fluid satisfying the strong energy condition. The quantum versions of such wormholes are consistent with the Hawking-Page conjecture for quantum wormholes as solutions of the Whecler-1)eWitt cquation. We contrast this with a classical change of signature approach which, although might be consistent with the existence of classical wormboles for a given definition of the energy-momentum tensor of the fluid, upon quantization gives everywhere oscillatory wavefunctions which do not satisfy the HP conjecture.

\section{Introduction}

One possible solution to the cosmological constant $(A)$ problem that has attracter a lot of interest is due to the idea that wormhole solutions can lead $\Lambda$ to become a dynamical variable with a distribution function $P(\Lambda)[1]$ - for a review of this proposal see, e.g., Ref. [2]. It is suggested that this function is peaked. due to De Sitter instantons, with the Baum-Coleman-Hawking factor $P(\Lambda) \sim t x p(1 / \Lambda)$, so predicting $\Lambda \rightarrow 0$ [1]-[3]-[4]. Wormholes are used in two distinct ways in such arguments. Firstly they are used to justify why $A$ should be treated as a dynamical quantum variable instead of a usual classical variable. This is the most important aspect, as it allows one to make predictions of the possible values of $\Lambda$. Wormboles have further been used in connecting many universes together, which produces a further exponentiation [1], i.e.

$$
I(\Lambda) \sim \exp (\exp (1 / \Lambda))
$$

This is only useful if the first factor $\sim 1 / \Lambda$ is correct (see, e.g. Ref. [5]). In other words, this aspect of wormholes only exaggerates any underlying behaviour.

In order that wormholes play a role, it is important that they should occur for fairly general matter sources. However, classical wormholes occur when the Ricri tensor has a negative eigenvalue: this is a necessary but not sufficient condition for their existence [6]. This condition is fairly restrictive, for example it excludes real scalar fields from being a suitable matter source for wormloles.

In simple lriedmann-Robertson-Walker (FRW) models, wormholes are typically described by a constraint equation of the form

$$
\frac{\dot{a}^{2}}{a^{2}}=\frac{1}{a^{2}}-\frac{\text { const }}{a^{n}}
$$

where $a$ is the scale factor in the (spatially closed) line etement $d s^{2}=d t^{2}+a^{2} d \Omega_{3}^{2}$, and the derivative is with respect to the Euclidean time $t$.

In order to have an asymptotically Euclidean ( $\mathrm{AF}$ ) wormhole it is necessary that $\dot{a}^{2}$ remains positive at large $a$, and this requires $n>2$. This wormhole represents two separate Euclidean regions connected together by a throat where $\dot{a}=0$, and the throat size $a_{0}$ is finite. Typical cases ase $n=6$ (axion) and $n=4$ (conformal scalar field). Using the conformal ime $\tau$ defined by $d t=a d \tau$, the typical solution of exp. (2) is

$$
a^{n / 2-1} \sim(\text { const })^{1 / 2} \cosh \left[\frac{(n-2)}{2} \tau\right]
$$


One sees the typical wormhole shape, e.g. $a \sim \cosh (\tau)$ for the conformal scalar $(n=4)$ case. If a cosmological constant was included it would dominate for large a giving, in total, a "dumb-bell" shape.

\section{Carlini-Mijić wormholes}

Carlini and Mijić [7] expanded the number of possible wormhole solutions by consid ering an analytic continuation of closed FRW universes. ${ }^{4}$ For a perfect fluid equation of state

$$
p=(\gamma-1) \rho
$$

(where $p$ and $\rho$ are, respectively, the pressure and energy density of the fluid). closed universes require that the strong energy condition be satisfied, i.e. that $\gamma>2 / 3$. It is the continuation of this theory to the Euclidean domain which then gives wormhole solutions. To sec this one can consider the Friedmann equation in Euclidean space

$$
\frac{\dot{a}^{2}}{a^{2}}=\frac{1}{a^{2}}-\rho
$$

The conservation equation for $\rho$ in the Lorentzian region is given by

$$
\dot{\rho}+3(\rho+p) \frac{\dot{a}}{a}=0
$$

and with the usual equation of state $p=(\gamma-1) \rho$ it has solution $\rho=\rho_{0} / a^{3 \gamma}$. This solution also occurs in Euclidean space if the equation of state (4) and the conservation eq. (6) hold the same in Eucliclean space (after the analytic continuation of $t \rightarrow i t$ ).

Substituting for $\rho$ into the Friedmann equation (5) gives

$$
\frac{a^{2}}{a^{2}}=\frac{1}{a^{2}}-\frac{\rho_{0}}{a^{3 \gamma}}
$$

which now has the form of the wormhole equation (2), with the simple correspondence $3 \gamma=n$. Wormholes are therefore possible for any $\gamma>2 / 3$ (i.e. when the strong energy condition is satisfied) which is a generalization of the previously found axion $(\gamma=2)$ and conformal scalar $(\gamma=4 / 3)$ cases. One can also easily evaluate the Ricci tensor from the Einstein equations, with the energy-momentum tensor for the fluid given by $T_{a b}=(\rho+p) U_{a} U_{b}+p g_{a b}$ (where $U_{a}$ is the four velocity of the fluid, such that $U_{a} U^{a}= \pm 1$ in the Euclidean and Lorentzian regions, respcclively, and $g_{a t}$ is

\footnotetext{
${ }^{4} \mathrm{~A}$ similar idea can also be found in Ref. [8].
}

the spatially closed FRW metrie). The result, valid both in the Euclidean and the Lorentzian regions, is that $R_{0}^{0}=(\underline{2}-3 \gamma) \rho / 2$, and clearly only for $)>2 / 3$ can the Ricci tensor have a negative eigenvalue and allow for the possibility of wormholes, $c f$ Ref. [6].

\section{Scalar field extension}

Lsing an adaptation of the work of Fllis and Madsen [9], we can use a scalar fick which simulates a certain value of $\gamma$. In Lorentzian space such a realization recpuires a potential of the form $[i]$

$$
V(\phi)=\frac{(2-\gamma)}{2 a_{0}^{2}} \cosh \frac{(3 \gamma-2)}{\sqrt{2} \gamma} \phi
$$

where $a_{0}$ is an arbitrary constant which represents the maxinum size of the closed universe for $\gamma>2 / 3$. In order to obtain a classical wormhole with this potential it is necessary to also analytically continue the scalar ficld to an imaginary value, i.c. $\phi \rightarrow i \phi,{ }^{5}$ so that the Euclicleau potential will have a $\cos$ form and $a_{0}$ becomes the throat size of the corresponding wormhole.

Again one finds that only for $?>2 / 3$ can a classical wormhole solution be obtained.

For later use, we remart that provided the classical equations are valid the scalar potential $V(\phi)$ can be re-written in terms of the scale factor $a$, such that

$$
V(\phi) \equiv V(a)=\frac{V_{m}}{a^{3 \gamma}}
$$

where the constant $V_{m}=(1-\gamma / 2) a_{0}^{3 \gamma-2}$.

\section{Quantum wormholes}

In a different vein, because the number of known wormhole solutions had appeared so limited, Hawking and Page (HP) considered that solutions of the Whecler-DeWitt (WDW) equation could more generally represent wormholes [10]. For such wormholes, they suggested that the guantum mechanical wavefunction $\Psi$ should decay exponentially for large scale factor $a$, so as to represent Euclidean space, and that $\Psi$ be well behaved as $a \rightarrow 0$, so that no singularities are present.

5Strickly speaking. tlis analytic continuation is 'asymmetric' with respect to that for the time
$t \rightarrow$ it, cf. Carlini in Ref. [i]. 
Because the WDW equation is independent of the lapse chosen, the Enclidean regime is already 'included' in the formalism. Thus, the quantum versions of the Carlini-Mijić (CM) wormholes should be found with no need of any explicit continuation.

Quantization of the constraint equation (7) gives the WDW equation (ser, f.g. Ref. [11])

$$
\left(a^{2} \frac{d^{2}}{d a^{2}}+q a \frac{d}{d a}+\rho_{0} a^{6-3 \gamma}-a^{4}\right) \Psi(a)=0
$$

where $q$ represents part of the factor ordering ambiguities.

We can get some iclea as to when an Euclidean domain occurs at large a by considering the sign of the potential $l(a)$ in the analogous cquation (setting the unimportant, in this regard, factor $q=0$ )

$$
\left(\frac{d^{2}}{d a^{2}}+l^{\prime \prime}(a)\right) \Psi(a)=0
$$

When $U>0$, oscillating solutions occur which represent Lorentzian metrics. 'There fore, in order to obtain a wormhole (an $\mathrm{AE}$ regime for large scale factor) we require $U<0$. Returning to our eq. (10), this occurs for

$$
I \equiv \rho_{0} a^{4-3 \gamma}-a^{2}<0
$$

Therefore, for the usual case of positive energy density $\left(\rho_{0}>0\right)$, we require $2>4-3-$ i.e. $\gamma>2 / 3$, for such behaviour (see Fig. 1).

This strong energy condition is the same as that obtained by ( $\mathrm{N}$ for the occurrence of wormhole solutions. Kin and Page [12] found that quantum wormholes are incontpatible with a cosmological constant. However we have here a stronger condition: perfect fluid matter sources violating the strong energy condition are incompatible with wormholes obeying the HP conditions. The presence of any matter soure with $\gamma<2 / 3$ will eventually dominate for large $a$ and prevent the AE: wormhole.

A quantum wormhole also requires suitable behaviour for small $a$. As $a \rightarrow 0 \mathrm{we}$ can ignore the $a^{4}$ term, since $4>0-3 \gamma$ when $\gamma>2 / 3$. In this case the WDW equation ( 10 ) (for $q=1$ and $\gamma \neq 2$ ) simplifies to a Bessel equation with solution

$$
\Psi(a) \simeq c_{1} J_{0}\left(\frac{2 \sqrt{\rho_{0}}}{3(2-\gamma)} a^{3-3 \gamma / 2}\right)+c_{2} Y_{0}\left(\frac{2 \sqrt{\rho_{0}}}{3(2-\gamma)} a^{3-3 \gamma / 2}\right)
$$

In order to satisfy the wormhole boundary condition we should choose the $J$ Bessel function. For the particular case $\gamma=2$, the solution of eq. (10) is a linear combination of Bessel functions, $J_{ \pm i \sqrt{B_{0}} / 2}\left(i a^{2} / 2\right)$, which oscillates an infinite number of times at the origin (see Ref. [10]), and therefore cannot satisfy the required regularity condition of HP.

Although the WDW is an ordinary differential equation, it is still not straightforward to obtain analytic solutions for all $\gamma$. Instead of proceeding by finding approxi mate WKB solutions, we can still emphasize important properties that any solution in the range $2 / 3<\gamma<2$ will have ${ }^{6}$ by considering the exactly soluble case of $\gamma=4 / 3$. This is the case of radiation (equivalent to a conformally coupled scalar field), which allows eq. (10), for $q=0$, to be written as (cf. Ref. [1.3])

$$
\left(\frac{d^{2}}{d a^{2}}+\rho_{0}-a^{2}\right) \Psi(a)=0
$$

This is in the form of a parabolic cylinder exuation with solutions in terns of confluent hypergeometric functions (see, e.g., Ref. [14])

$$
\begin{aligned}
\Psi(a) & \simeq a^{2} p\left(-a^{2} / 2\right)\left[c_{3} \cdot{ }_{1} F_{1}\left(1 / 4\left(1-\rho_{0}\right) ; 1 / 2: a^{2}\right)\right. \\
& \left.+c_{4} \cdot{ }_{1} F_{1}\left(1 / 4\left(3-\rho_{0}\right) ; 3 / 2 ; a^{2}\right) \cdot a\right]
\end{aligned}
$$

For $\rho_{0}>1$ and $c_{4}=0$ we get a regular oscillation for small " with the Fucliclean regime for large a, see Fig. (2).

We should point out that throughout this section we ate only interested in the existence of wormhole solutions, and set arbitrary coefficients arcordingly. In theory such coefficients should be determined by the boundary conditions applied. The usually applied no-boundary conditions of Hartle-Itawking [15] or the Tunneling one (see Ref. [16]), would in general contradict such solutions [17]

\section{Scalar field extension}

If wormholes could only occur when the strong energy condition was satisfied, they would still seem rather restrictive. However, working with the scalar field model with potential $V(\phi)$ given by $c$. (s), quantum wormholes can be obtained even when the strong energy condition is violated, except for the cosmological constant $\gamma=0$ case [17]. These wormholes can satisfy the HP conditions up to an arbitrarily large size, but they are somewhat unsatisfactory in that, roughly speaking, matter components

${ }^{6}$ The upper boundary $\gamma \leq 2$ is dictated by the requirement that the sound wave velocity of the fluid must be smaller tlian the speed of light.

'Note that the asymmetric continuation required for wormholes is 'included' in the formalism cf. Linde in Ref. [16] 
have small values in relation to generic separation const ants. They also suffer from not being consistent with the more usual boundary conditions which predict inflationary behaviour (for technical details and further discussion see Ref. [17]).

\section{Classical signature change}

There is, however, a different approach based on 'classical change of signature' [18]. Now the metric for a FRW universe is taken to be of the form

$$
d s^{2}=-\epsilon d t^{2}+a^{2}\left[\frac{d r^{2}}{1-k r^{2}}+r^{2} d \Omega_{2}^{2}\right]
$$

Here $\epsilon$ represents the signature change: $\epsilon=1$ for usual Lorentzian space and $\epsilon=-1$ in Euclidean space. The three dimensional spacelike sections at $t=$ const are flat. open or closed if $k=0,-1$ or +1 , respectively.

We first consider a perfect fluid with energy-momentum tensor defined as [18]

$$
T_{a b}=(\rho+\epsilon p) U_{a} U_{b}+p g_{a b}
$$

where, as usual, $U_{a}$ is the four velocity of the fluid and $g_{a b}$ is the metric of eq. (16). The Friedmann equation turns out to be (cf. Ref. [18])

$$
\frac{\dot{a}^{2}}{a^{2}}=\rho-\frac{\epsilon k}{a^{2}}
$$

and the energy conservation equation is

$$
\dot{\rho}+3(\rho+\tau) \frac{\dot{a}}{a}=0
$$

The idea of classical signature change is that $\dot{a}^{2} / a^{2}$ should remain positive so that the need of analytic continuation to imaginary time is excluded. This provides a criteria for when the signature change occurs (for $k \neq 0$ ): $\epsilon=\operatorname{sign}(k)$ when $\rho>1 / a^{2}$. while $\epsilon=-\operatorname{sign}(k)$ when $\rho<1 / a^{2}$.

However, in order that the junction conditions be satisfied (that the scale factor and the energy density are continuous through the surface $\Sigma$ of change of signature). one must have $k=0$ (see Ref. [18]). The matter source, from the outset, cannot allow for the possibility of a wormhole.

If we proceed regardless and anyway let $k \neq 0$, then the WDW equation corresponding to the Friedmann eq. (18) can be readily obtained following the methods of Martin [19] (sce also Ref. [20]) ${ }^{*}$

$$
\left(a^{2} \frac{d^{2}}{d a^{2}}+q a \frac{d}{d a}+\rho a^{6}-k \in a^{4}\right) \Psi(a)=0
$$

Substituting from the conservation equation for $\rho$ (for the case of $\rho$ continuous and with unique functional form in the Euclidean and Lorentzian manifolds), gives the WDW potential (cf. eq. (12))

$$
l^{\prime} \equiv \rho_{0} a^{4-3 \gamma}-k \in a^{2}
$$

We can ensure (for the case $k=1$ ), that the change of signature will let $U$ remain positive. This will give purely oscillatory wavefunctions, and no instanton-like ('classically forbidden') behaviour of the form $\sim \exp (-S)$ will be present."

The weakness in the previous arguments (that, really we should lave set $k:=0$ ) cant also be seen by looking at the WDW potential: in order that the WI)W can have well defined (i.e. ( ${ }^{2}$ differentiable) solutions, the potential $t /$ of eq. (21) should be continuous, thus requiring $k=0$.

\section{Scalar field extension}

However we can remedy this by using a scalar ficld where the junction conditions require $k=1$. Essentially a similar result (of having purely oscillatory wasefunctions) will be obtained, which we briefly sketch.

The Friedmann equation now has the form

$$
\frac{a^{2}}{a^{2}}=\dot{\phi}^{2}+t\left(V(\phi)-\frac{k}{a^{2}}\right)
$$

We consider the scalar field model with potential given by oq. (8) which simulates a certain value of $\gamma$

We can simplify the analysis further by substituting the classically equivalent potential $V(a)$ into the Friedmann equation (22). In doing so. i.e. assuming the existence of the fixed relationship (9) bet ween $\phi$ and $a$, we are in some sense effectively 'freezing out' the scalar field degree of freedom, yet this toy model still enables us to show how the main properties of the model arise.

\footnotetext{
${ }^{8}$ Note that, althongh the idea of classical signature cliange is that Fuclidean regions can be 'In pro equations can in turn lie quantized

'In principle, one could he 'perverse' and choose the signature change to keep $a^{2} / a^{2}$ negative, so ensuring an $\sim \exp (-S)$ type behaviour everywhere. But this contradicts the original motivation of
classical signat ure cliange.
} 
The WDW equation obtained from this modified Friedmann equation will have the same form as eq. (20), but (for $k=1$ ) with the potential (cf. Ref. [19])

$$
U=\epsilon\left(V_{m} a^{4-3 \gamma}-a^{2}\right)
$$

To get oscillatory wavefunctions requires $U>0$, which is achieved by use of the signature change $\epsilon$ parameter.

The choice $\gamma<2 / 3$ seems more physically reasonable in this model. In such cases the usually forbidden region at small scale factors becomes classically allowed by taking $\epsilon=-1$. This means that $U$ remains positive and the wavefunctions remain oscillatory even for small scale factors. This case agrees with the original motivation of classical signature change which was to avoid the need for Fucliclean time during the early and presumaloly small universe [18].

On the other hand, for $\gamma>2 / 3$ a classical signature change would allow the existence of a classical description beyond the usual maximum size of a closed miverse. This appears to be suspect on physical grounds, especially if we werr dealing with large universes, but it might be justified by considering such closed universes to be of order of the Planck size. If so, then the resulting signature change will give oscillatory wavefunction beyond their maximum size, so preventing the $\mathrm{HP}$ conditions being satisfied, and the existence of quantum wormholes, in such a region.

Analogous results could have been obtained using other potentials $l(\phi)$ witlout resorting to the $V(a)$ potential (for instance, one could expand the scalar field poten tial for small values of $\phi$ and get an effective cosmological constant. of. Ref. [19]). but the $\phi$ dependence would have been, of course, more complicated.

\section{Changing $T_{a}$}

At this stage, we should point out that there is still some frectom in defining the energy monentum tensor for the perfect fluid: the main requirments are expected to be 'smoothness' of $T_{\mathrm{at}}$ at the junction surface $\Sigma$ and agreement with its standard expression in the Lorentzian region. Besides the [18] choice, another possib]le trusor is provided by the expression

$$
T_{a b}=\epsilon(\rho+p) U_{a} U_{b}+p g_{a b}
$$

whose main property is to have $-\rho, p, p, p$ as eigenvalues in both the Euclidean and Lorentzian signature regions.
Repeating the calculations leading to ecss. (18)-(19), one can easily check that the Friedmann equation for a FRIV nuiverse with the matter content of eq. (24) becomes

$$
\frac{a^{2}}{a^{2}}=\epsilon\left(\rho-\frac{k}{a^{2}}\right)
$$

while the energy conservation equation is given again by the (standard) equation (6) (which is the same in both signature regions).

This time, positivity and continuity of $\dot{a}^{2} / a^{2}$ can be easily seen to be guarantecd by the condition that $\left.\rho\right|_{\Sigma}=\left.\left(k / a^{2}\right)\right|_{\Sigma}$ at the junction surface $\left(\left.a\right|_{\Sigma} \doteq a_{0}\right)$, which is perfectly consistent with the existence of a closed universe $(k=1)$ (provided that, as usual, $\rho>0$ ). In other words, the extrinsic curvature (and $\dot{a}$ ) must be zero on $\Sigma$, and the change of signature is of the strong type [20]. Furthermore, for $k=1$, the signature will be Lorentzian $(\epsilon=1)$ when $\rho>1 / a^{2}$, and Euclidean $(c=-1)$ when $\rho<1 / a^{2}$.

In particular, for $p, \rho$ and the equation of state (4) continuous, the solution of the conservation eq. ( 6 ) is given by $\rho=\rho_{0} / a^{3 \gamma}$ (for both signatures). Consequently the Friedmann e(p. (25) in Euclidean space is again exactly of the form (2), and one has the same Euclidean wormhole solutions (with throat size $a_{0}$ ) as in the CM case, i.e. when the strong energy condition is satisfied. ${ }^{10}$ Mforeover. the solution of the same Friedmann equation in Lorentzian space clearly represents a closed and expranding FRW universe with maximun size $a_{0}$. What the change of signature approash is suggesting in this case, is that in principle one conld have a change of signature also at large scale factor, and that the 'continuation' of the closed universe at its maximum size is an $\mathrm{AF}$ wormliole. The difference with respect to the standard CM approach is that, in the context of the classical signature change model, there is no necd of resorting to any coutinuation into imaginary time.

We can then cuantize in a similar fashion the constraint e(f. (25) for the tensor (24) to obtain the following WDW equation (cf. Ref. [19])

$$
\left[a^{2} \frac{d^{2}}{d a^{2}}+q a \frac{d}{d a}+\epsilon\left(\rho a^{6}-k \cdot a^{4}\right)\right] \Psi(a)=0 .
$$

where now, substituting from the conservation equation for $\rho$ (and with $q=0$ ), the potential $U$ is given by

$$
U \equiv \epsilon\left(\rho_{0} a^{4-3 \gamma}-k a^{2}\right)
$$

${ }^{10}$ Evaluating, as in the ( $\mathrm{M}$ case, the Ricci tensor from the Vinstein equations and witl the energy-momentum tensor (24). gives once more $R_{0}^{0}=(2-3 \gamma) \rho / 2$, which is negative for $\gamma>2 / 3$. 
Also in this case, as for when the matter content was clescribed by ca. (17), the classical change of signature picture requires that we choose $\epsilon$ such that $l$ will be positive every where (this time $U$ is continuous, provided the classical junction condi tions hold). This will give purely oscillatory wavefunctions everywhere, which do not satisfy the HP conditions for the existence of quantum wormholes.

This result is not modified by the extension to a scalar field model with the potential simulating a certain value of $\gamma .{ }^{11}$

\section{Conclusions}

Using a perfect fluid matter source we have found that classical wormhole solutions (obtained by an analytic continuation) occur only when the strong energy condition is satisfied. The quantum analogues of such wormholes agree with the HP conjecture for conditions to be satisficd by quantum wormholes.

We have briefly mentioned that quantum wormholes (but not classical ones) can also be obtained for violations of the strong energy condition if a scalar field matter source is used. The scalar potential $V(\phi)$, obtained by Carlini and Mijic, that in a sense simulates a perfect fluid with a certain value of $\gamma$, can be used for such a purpose. These wormholes suffer from a number of limitations, among them that they contradict other requirements that are equally useful (see Ref. [17] for further details).

The change of signature approach tries to entirely avoid the presence of classically forbidden regions in which the wave function would usually behave exponentially (the HP conditions). Although this proposal has some foundation when the forbidlen region is at small scale, it is difficult to justify when the forbidden region is beyond a Lorentzian small scale description. It therefore seems more appropriate. in the first model considered, to only have classical signature change when the strong tnergy condition is violated.

A similar classical change of signature approach, based on a morlified energymomentum tensor for the perfect fluid, can instead reproduce the classical (M solutions for a closed Lorentzian expanding universe $(\gamma>2 / 3)$ which is 'continued' at its maximum radius into an $\mathrm{AE}$ wormhole, but with no need of any imaginary time. At by $V(a)$ particular, the analytic form of $V(\phi)$ will be the same as that given by eq. (8) and. replacing by $V(a)$ from eq. (9), one will obtain the same WDW potential (23). the quantum level. howerer, the WDW wave functions are still oscillating everywhere and there are no guantum wormhole solutions in the sense of III'.

Although the classical signature change gives oscillatory wavefunctions, there is still need of an explanation of why one should choose $\dot{a}^{2}$ positive in eqs. (18), (22) and (25). As previously mentioned, one could choose the opposite condition and get. an "everywhere forbidden" behaviour (cl. Ref. [22], where it is suggested that there is some preponderance of Lorentzian over Euclidean behaviour, while oscillations between the two cases are constantly occuring). An understanding of this point is required before one could fully just ify the use of classical signat ure change in obviating the need for guantum wormbole behaviour.

The difference betwecn purely oscillatory and exponential wavefunctions will have a crucial effect on arguments setting $\Lambda \rightarrow 0$. Once the exponential peak is lost, one wonld expect wider predictions for $A$ if it is given by a clistribution function $P(A)$. Indeed, the classical signature clange approach seems only compatible with the tunneling boundary condition [19], which would give larger values of $A$ [21].

There are also a mumber of of her out standing questions before we call compare the applicability of classical signature clange viz-a-viz quantum wormbole behaviour. A proper consideration of the signature variable within a more general action principle (see. e.g., Refs. [20] and [2:3]) is still an interesting and open question which we hope to address in the future.

\section{Acknowledgements}

We would like to thank Prof. G.F.R. Fllis for his continued interest and mcouragenent during the course of this work. A.C.'s research is supported by an EEC: fellowship in the 'Iluman (apital and Mobility' program, under conlract No. ERBCHBICT:30:31:3. 


\section{References}

[1] S. Coleman, Nucl. Phys. B 310, 643, (1988),

[2] S. Weinberg, Rev. Mod. Phys. 61, 1, (1989).

[3] E. Baum, Phys. Lett. B 133, 185, (1983).

[4] S.W. Hawking, Phys. Lett. B 134, 403, (1983)

[5] J. Polchinsky, Phys. Lett. B 219, 251, (1989).

[6] S. Giddings and A. Strominger, Nucl. Phys. B 306, 890, (1988).

[7] A. Carlini and M. Mijić, Spacetime wormholes as analytic continuation of closed expanding universes, SISSA preprint 91 A (1991);

A. Carlini, Spacetime wormholes with a scalar field: a new set of exact solutions, SISSA preprint 65/92/A (1992);

A. Carlini and M. Martellini, Class. Quantum Grav. 9, 629, (1992).

[8] A. Zhuk, Phys. Lett. A 176, 176, (1993).

[9] G.F.R. Ellis and M.S. Madsen, Class. Quantum Grav. 8, 667, (1991)

[10] S.W. Hawking and D.N. Page, Plyys. Rev. D 42, 2655, (1990).

[11] M. Ryan, Hamiltonian Cosmology, (Springer-Verlag, Berlin, 1972): B.S. DeWitt, Phys. Rev. 160, 1113, (1967),

[12] S.P. Kim and D. N. Page, Phys. Rev. D 45, R3296, (1992).

[13] P.F. Gonzalez-Diaz, Mod. Phys. Lett. A 5, 1307, (1990).

[14] M. Abramowitz and I.A. Stegun, Handbook of Mathematical Functions (Dover, New York, 1965)

[15] J.B. Hartle and S.W. Hawking, Phys. Rev. D 28, 2960, (1983)

[16] A. Vilenkin, Phys. Rev. D 30, 549, (1984);

A.D. Linde, Sov. Phys. JETP 60, 211, (1984);

V.A. Rubakov, Phys. Lett. B 148, 280, (1984);

Y.B. Zeldovich and A.A. Starobinski, Sov. Astron. Lett., 135, (1984).
[17] A. Carlini. D.H. Coule and D.M. Solomons, Quantum versions of CarlinzMijić wormholes, NORDITA preprint NORDITA-94/75 A, bulletin board grqc/9412042, (1994).

[18] G.F.R. Ellis, A. Sumeruk, D. Coule and C. Hellaby, Class. Quantum Grav. 9, 1535, (1992); G.F.R. Ellis, Gen. Relativ. Gravit. 24, 1047, (1992).

[19] J. Martin, Phys. Rev. D 49, 5086, (1994)

[20] F. Embacher, Actions for signature change, University of Vienna preprint UWTHPH-1995-1, bulletin board gr-qc/9501004, (1995).

[21] D.H. Coule, Quantum cosmology and the value of $\Lambda$, University of Cape Town preprint, bulletin board gr-qc/9408024, (1994).

[22] F. Embacher, Signature change induces compactification. University of Vienna preprint UWTHPH-1994-47, bulletin board gr-qc/9410012 (1994).

[23] J. Greensite, Phys. Lett. B 300, 34, (1993);

A. Carlini and J. Greensite, Phys. Rev. D 49, 866, (1994). 


\section{Figure Captions}

Fig. 1 The Wheeler-DeWitt potential $U(a)$ (with $\rho_{0}=1$ ). The Euclidean regime has $U<0$.

a) the case $\gamma=0$

b) the case $\gamma=4 / 3$

Fig. 2 An example of a quantum wormhole solution for the case $\gamma=4 / 3$ (eq. (15) with $c_{4}=0$ and $\rho_{0}=25$ ).

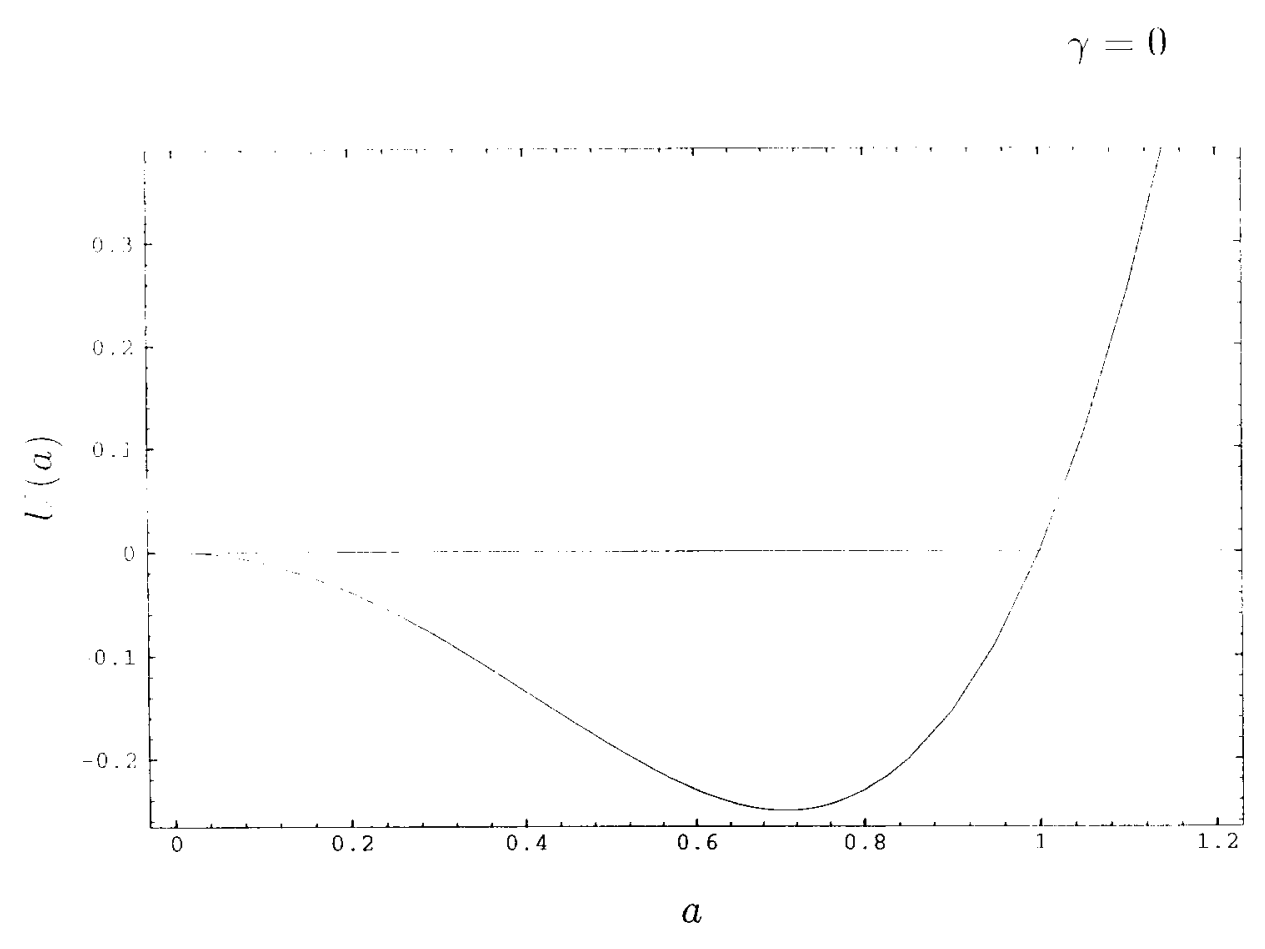

Fig. 1a 
$\gamma=4 / 3$

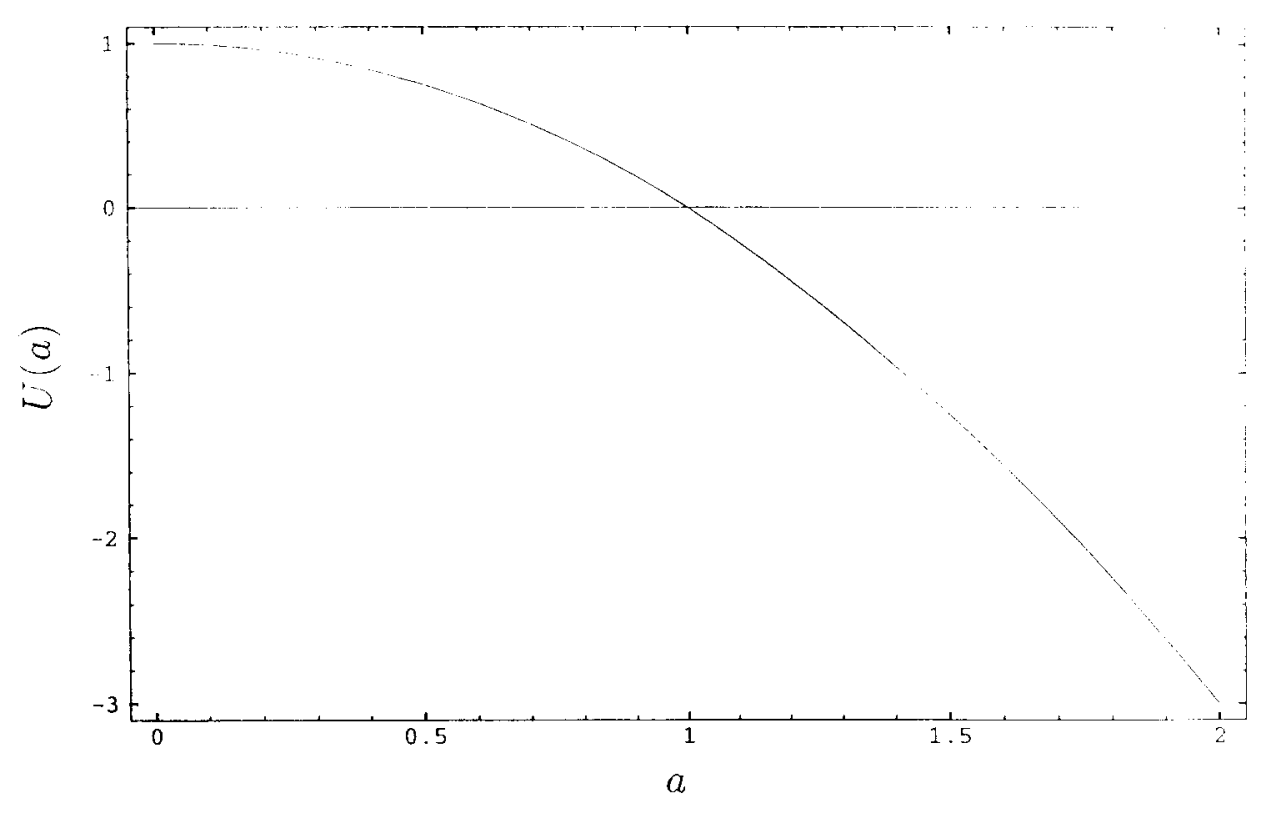

Fig. 1b

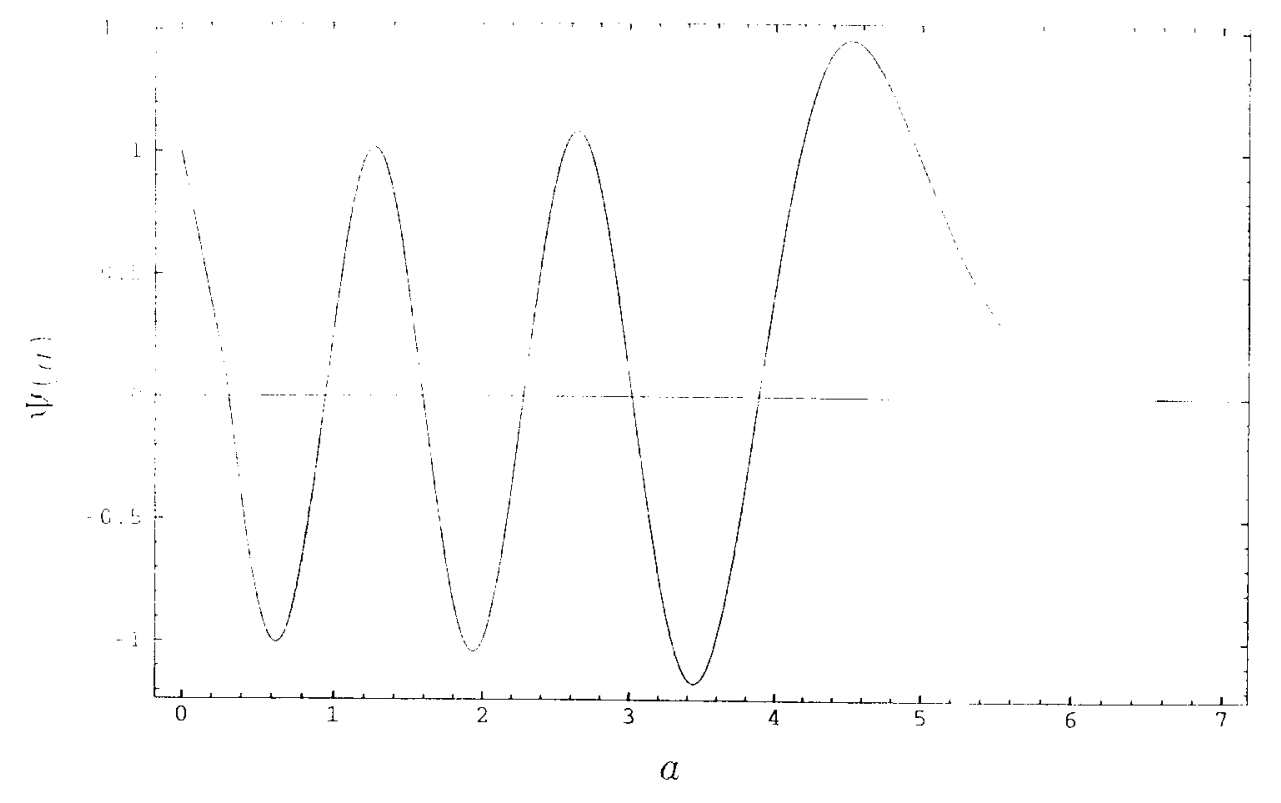

Fig. 2 
